\title{
The Comparative Study on Machining Deformation and Experiment of Integrated Frame Part
}

\author{
Dong Zhao-wei ${ }^{1,}$, , Wan Xiao-hang ${ }^{2, b}$, Liu Sheng-yong ${ }^{3, c}$ \\ ${ }^{1}$ Hebei University of Economics and Business, Shijiazhuang, 050061, China \\ ${ }^{2}$ Hebei College Industry and Technology, Shijiazhuang, 050091, China \\ ${ }^{3}$ School of Mechanic Engineering, Hebei Normal University, Shijiazhuang, 050031, China

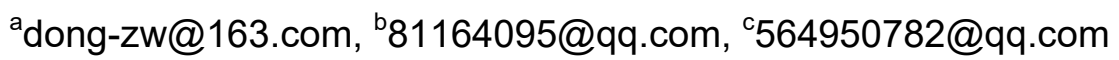

Keywords: Integrated frame; residual stress release; machining deformation; element life and death; cutting experiment.

\begin{abstract}
In aerospace equipment manufacturing, the machining deformation of the large integral structure is seriously influence on the machining efficiency and the accuracy of the parts. Based on the theory of elastic-plastic theory, the finite element analysis model of machining deformation of 3D integral frame is established, and the removal process of the material is simulated by using the technology of element birth and death. The deformation law of the initial residual stress release caused by the initial residual stress is studied, and the cutting experiment is designed, and the deformation of each milling is measured by using a three-dimensional coordinate measuring machine. Through the comparative analysis of the simulation data and the experimental data, it can make a conclusion that establishment of a correct analysis of the model can predict the machining deformation, and reduce the machining deformation.
\end{abstract}

\section{Introduction}

In aerospace equipment, more and more integrated structural parts instead of the traditional assembly structure, improve production efficiency and reduce the probability of accidents [1-2]. For example: integrated frame, wing, and other parts.[3-4] The features of this kind of parts are large size, complex structure, high machining accuracy, large machining allowance and poor rigidity of parts. The residual stress in the blank affects the machining accuracy of the parts, which often leads to the deformation of parts and seriously affects the production schedule[5-6].

It has been successfully applied to analyze machining deformation by using finite element theory, but most researches are two-dimensional cutting. In recent years, with the progress of computer technology and the improvement of finite element theory, it lays a foundation for the study of 3D Cutting [7-8]. This paper establishes the overall frame parts milling 3D finite element analysis model based on finite element theory, the "element birth and death" technique material removal process simulation analysis, obtained the release of residual stress caused by the deformation of parts of the design and cutting experiments using 3D coordinate measuring instrument for measuring the time of milling deformation. By comparing the simulation data with the experimental data, the analysis model is validated.

\section{Analysis Model}

The part drawing is shown in figure 1, The work-piece is positioned with the bottom plane as the locating surface, and the two ends are clamping positions. In order to improve the efficiency of analysis and reduce the time occupied by the computer hardware, half of the longitudinal part is analyzed. When the seed point is set and the mesh is finished, the outer surface of the part coincides with the boundary of the element. In this paper, a low order 8 node hexahedral element is used to divide the model into 188700 units and 200830 nodes.

The residual stress is firstly set up before simulation analysis. The number of parts according to the layered thickness direction, the discretization of the distribution curves of the stress test in the 
residual, in accordance with the "force balance and torque balance" principle, the initial residual stress loading on the model. Fig. 2 is the distribution of the 7075-T7351 aluminum alloy pre-stretched sheet by layer by layer cutting method in the depth direction [9], the elastic modulus of the material is $73 \mathrm{GPa}$, the Poisson's ratio is 0.35 , and the material density is $2780 \mathrm{Kg} / \mathrm{m}^{3}[10]$.

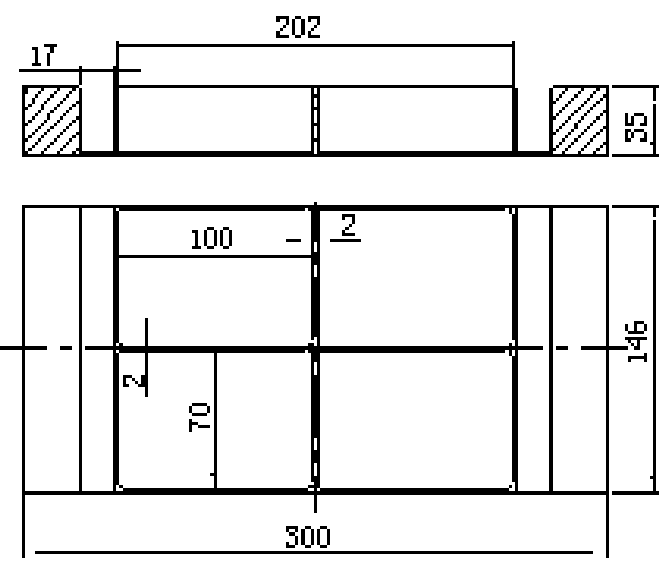

Fig.1 part dimension

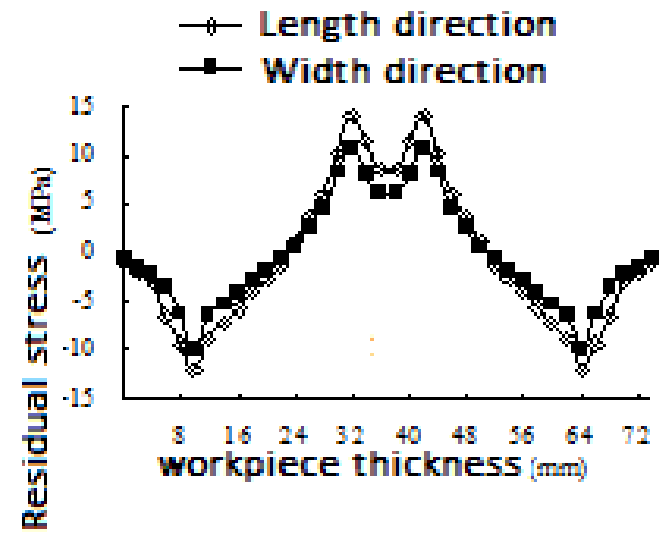

Fig. 2 residual stress distribution

\section{Milling Process Analysis}

The constraint condition of the model boundary condition is set up according to the clamping condition of the cutting experiment of the part. After each layer of machining simulation is completed, the deformation data of the part is obtained through the constraint transformation, and the steps are as follows: First remove cutting loads; and then release the displacement boundary condition; a point in the bottom parts non machining parts, limit three direction of rigid body displacement, while the other two parts of the bottom surface in different directions to limit rotational degrees of freedom, the free deformation of parts. In this paper, the cell life and death technology is adopted to simulate the removal process of materials. Unit to be "killed" in the post process is not visible, "die unit" is not removed from the model, but MARC software for element stiffness matrix multiplied by a factor of 10-6, the load associated with the "dead cell" is zero, and "dead cell" quality, damping, heat and other factors is zero, the element strain is zero. The analysis results are shown in figure 3 -figure 4 . From the figure we can draw deformation data, the cutting part for each layer, residual stress will release and redistribution of stress distribution will lead to deformation of parts, the parts material was removed to the side bending, bending degree with different cutting depth. 


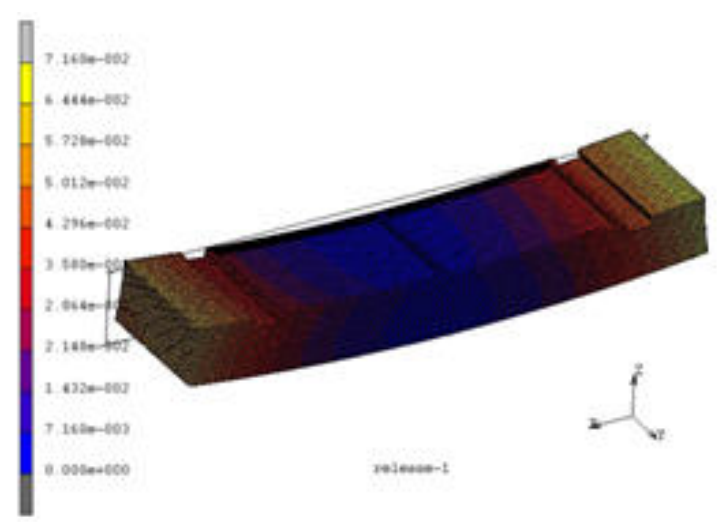

(a) $5 \mathrm{~mm}$ machining deformation

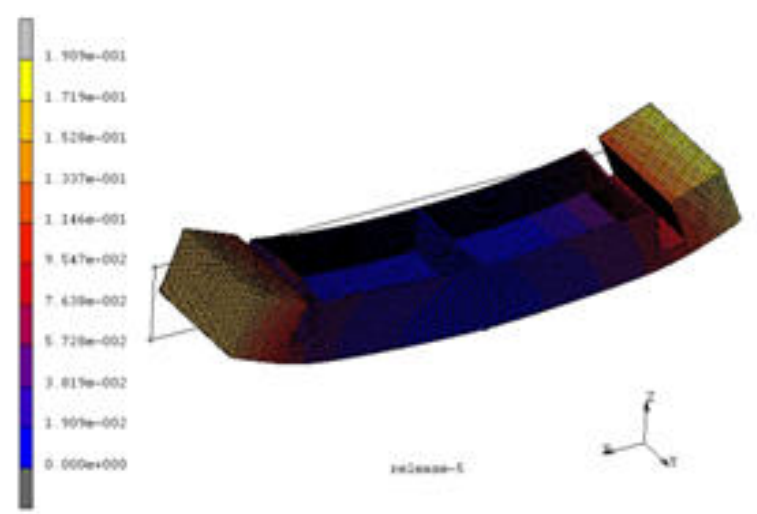

(c) $15 \mathrm{~mm}$ machining deformation

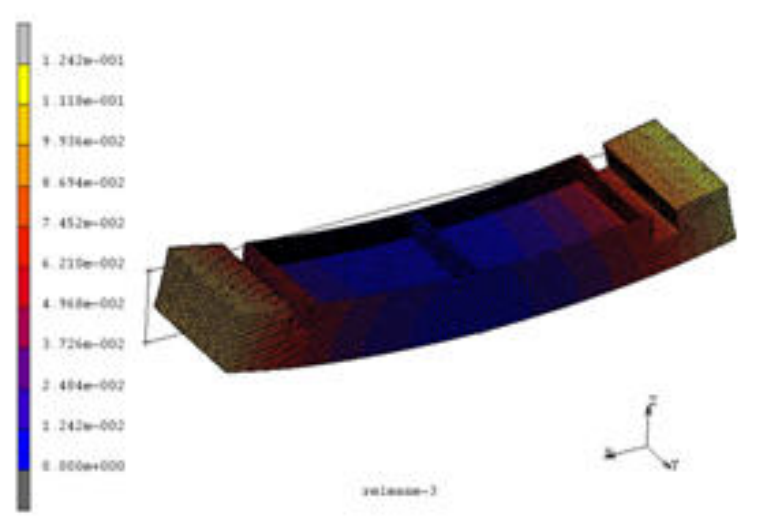

(e) $25 \mathrm{~mm}$ machining deformation

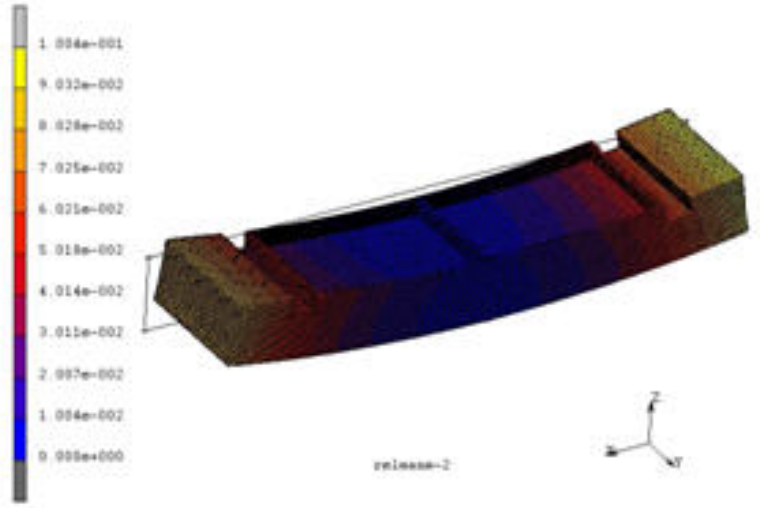

(b) $10 \mathrm{~mm}$ machining deformation

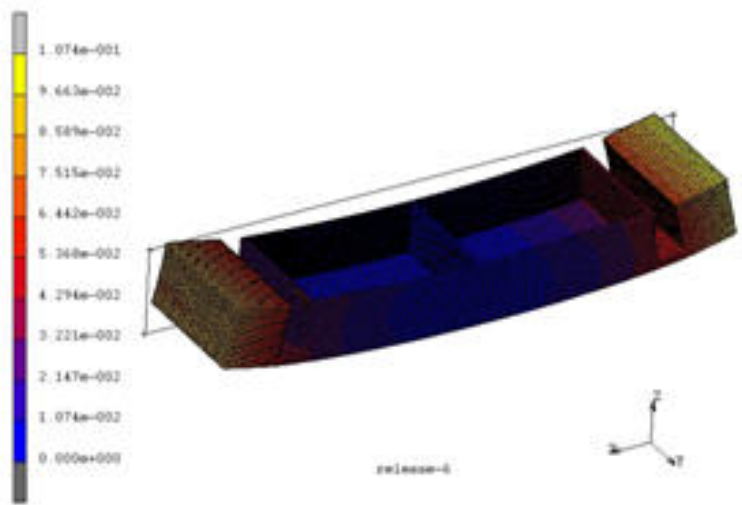

(d) $20 \mathrm{~mm}$ machining deformation

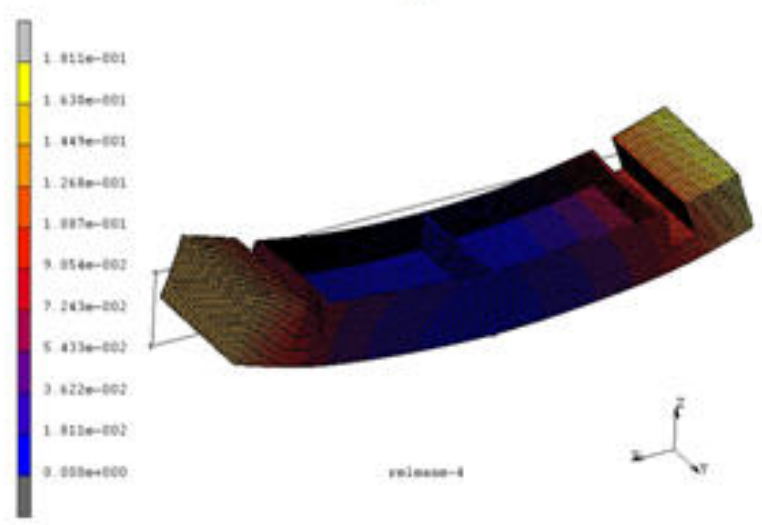

(f) $30 \mathrm{~mm}$ machining deformation

Fig.3 machining deformation

Fig. 4 is the finite element simulation of the deformation curve of the specimen. As can be seen from the diagram, the deformation in the first 4 layers of milling increases gradually, and when the milling depth reaches about $60 \%$, the deformation amount of the specimen reaches the maximum value. From the fifth layer milling, the deformation of the specimen decreases gradually. Because the milling depth is more than $60 \%$, the residual stress in the pre - removal part of the bottom of the specimen is tensile stress, so the deformation at both ends decreases gradually. It can be used for reference in the actual processing, when the milling depth reached $60 \%$ after machining, the work-piece from the fixture should be removed, the free deformation after keep free clamping, can greatly eliminate the work-piece deformation and subsequent processing. 


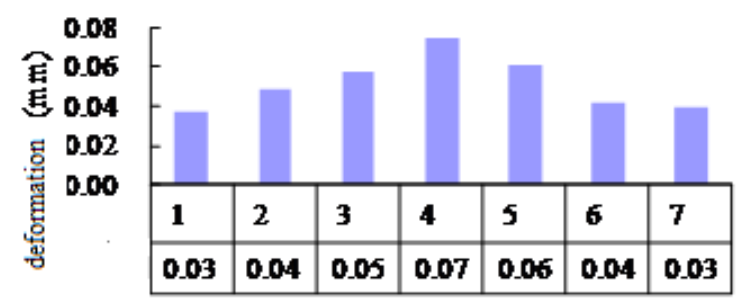

layer

Fig.4 simulation deformation analysis

\section{Data Comparison Analysis}

Experimental conditions: the diameter $10 \mathrm{~mm}$ carbide coated end mill, spindle speed4000rpm, feed F200mm/min, milling width of 5mm, using cutting fluid, as shown in Figure 5. Each of the milling depth after $5 \mathrm{~mm}$ (seventh times the milling depth is $3 \mathrm{~mm}$ ), remove the parts from the bench, using three coordinate measuring instrument for measuring work-piece set on the feature point deformation along the depth direction, as shown in Figure 6, the measurement data are shown in table 1. The data comparison is shown in figure 7.

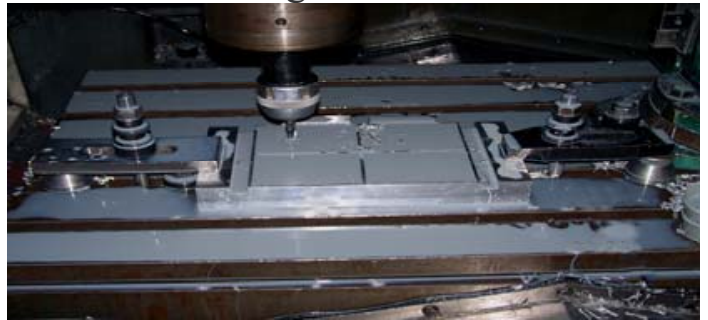

Fig.5cutting experiment

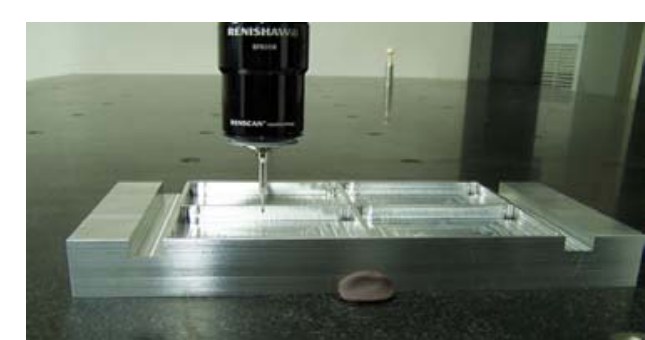

Fig.6 machining deformation measurement

According to the simulation results of the finite element machining and the measured results of the work-piece after cutting, the deformation tendency of the parts is the same. When the parts are machined to $60 \%$ of the thickness of the parts (fourth layers of milling), the processing deformation of the parts reaches the maximum value, and the deformation of the work-piece will gradually decrease when the work-piece is continued to be machined. When the cutting depth is more than $80 \%$ of the work-piece, the deformation of the work-piece changes little.

\begin{tabular}{|c|c|c|c|c|c|}
\hline Milling depth & $\frac{X}{Y}$ & 102 & 0 & -102 & planeness \\
\hline \multirow{3}{*}{5} & 72 & 0.013 & 0.011 & 0.011 & \multirow{3}{*}{0.018} \\
\hline & 0 & 0.009 & 0.001 & 0.005 & \\
\hline & -72 & 0.024 & 0.014 & 0.019 & \\
\hline \multirow{3}{*}{10} & 72 & 0.011 & 0.003 & 0.009 & \multirow{3}{*}{0.021} \\
\hline & 0 & 0.020 & 0.015 & 0.018 & \\
\hline & -72 & 0.029 & 0.016 & 0.025 & \\
\hline \multirow{3}{*}{15} & 72 & 0.026 & 0.017 & 0.018 & \multirow{3}{*}{0.026} \\
\hline & 0 & 0.020 & 0.007 & 0.011 & \\
\hline & -72 & 0.040 & 0.022 & 0.027 & \\
\hline \multirow{3}{*}{20} & 72 & 0.059 & 0.031 & 0.036 & \multirow{3}{*}{0.038} \\
\hline & 0 & 0.068 & 0.033 & 0.039 & \\
\hline & -72 & 0.089 & 0.046 & 0.048 & \\
\hline \multirow{3}{*}{25} & 72 & 0.059 & 0.031 & 0.036 & \multirow{3}{*}{0.044} \\
\hline & 0 & 0.068 & 0.033 & 0.039 & \\
\hline & -72 & 0.089 & 0.046 & 0.048 & \\
\hline \multirow{3}{*}{30} & 72 & 0.063 & 0.046 & 0.042 & \multirow{3}{*}{0.041} \\
\hline & 0 & 0.072 & 0.043 & 0.048 & \\
\hline & -72 & 0.094 & 0.051 & 0.062 & \\
\hline \multirow{3}{*}{33} & 72 & 0.088 & 0.086 & 0.089 & \multirow{3}{*}{0.028} \\
\hline & 0 & 0.081 & 0.072 & 0.088 & \\
\hline & -72 & 0.115 & 0.088 & 0.110 & \\
\hline
\end{tabular}




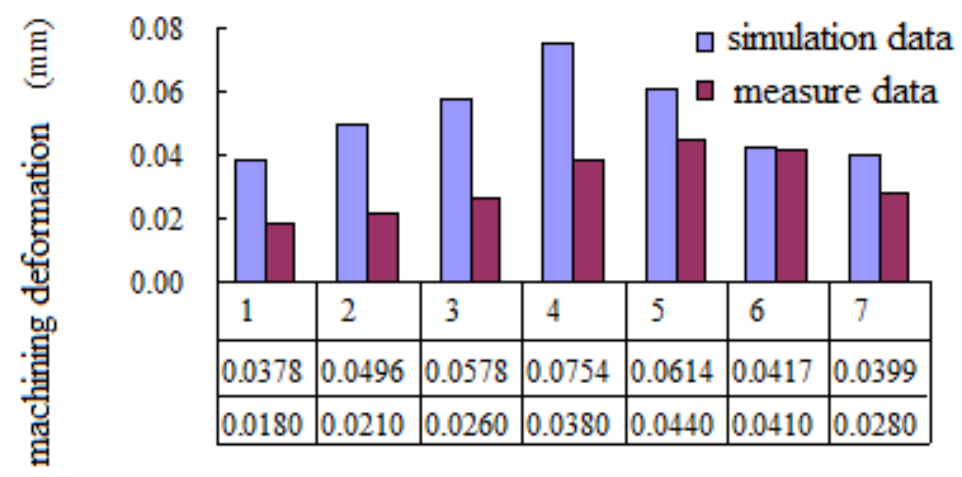

Fig.7 data comparative analysis

\section{Conclusion}

This paper establishes the overall frame parts milling 3D finite element analysis model based on finite element theory, the "element birth and death" technique material removal process simulation analysis, obtained the release of residual stress caused by the deformation of parts of the design and cutting experiments using 3D coordinate measuring instrument for measuring the time of milling deformation. Through the simulation and experiment data analysis, validate the analysis model, the overall structure of box parts machining deformation law, in order to develop is conducive to the rational processing technology provides the basis for reducing machining deformation.

\section{References}

[1] Liu shengyong, Dong zhaowei. study on machining deformation of milling because of part structure type[J]. machinery design and maunfacture. 2007(3):103-105.

[2]Kong xiao, Li ming, Bian dachao, Machining deformation control technology of aluminum alloy thin wall parts[J]. Machinery design and maunfacture. 2010(2):246-247.

[3] Gang Fang, Pan Zeng. Three-dimensional thermo-elastic-plastic coupled FEM simulations for metal oblique cutting processes[J]. Journal of Materials processing Technology, 2005,168:42-48

[4] Jacobus K, Kapoor S G, Devor R E. Experimentation on the Residual Stresses Generated by End-milling[ J]. Journal of Manufacturing Science and Engineering, 2001, 123:748-752.

[5]Zhao lili,Zhang yidu. the residual stress in the pre tension plate rolling computer simulation[J]. Journal of Beihang University.2004,30(7):606-609.

[6] Dong Hui Yue, Ke Yinglin. A Simulation of 3D Chip Shaping of Aluminum Alloy 7075 in Milling Processes [J]. Transactions o f Nonferrous Metals Society of China, 2005, 6( 12):1315-1321.

[7] Lu ying, Fan shiping,Bao yongjie, Experimental study on the deformation of high speed milling of thin wall parts of aviation aluminum alloy[J]. Journal of Shenyang University. 2012(3):28-30.

[8] Tang zhitao, Liu zhanqiang,Ai xing, Study on the residual stress of aluminum alloy in high speed milling process[J]. China Mechanical Engineering.2008(3):699-701.

[9]Dong zhaowei, study on the integrated framework machining residual stress and machining deformation[D]. Beihang University doctor degree thesis. 2006,89-94.

[10] Beijing Institute of Aeronautical Materials, aeronautical materials science[M]. Shanghai science and technology press, 1985:145-146. 